\title{
Influence of pneumococcal conjugate vaccine 13 on upper respiratory tract microbial biodiversity in infants
}

\author{
SAMAT KOZHAKHMETOV ${ }^{1,2,6, \vartheta}$, YERMEK AITENOV ${ }^{1}$, BAKHYT RAMAZANOVA, \\ YEKATERINA KOLOSKOVA ${ }^{3}$, TATYANA BURKUTBAYEVA ${ }^{4}$, LYAZZAT YERALIYEVA $^{5}$, \\ KAMILYA MUSTAFINA ${ }^{3}$, GULSHAKHAR BEGLAROVA ${ }^{7}$, MARAL YERGAZINA ${ }^{7}$, \\ ALMAGUL KUSHUGULOVA ${ }^{1,2}$ \\ ${ }^{1}$ Laboratory of Human Microbiome and Longevity, Center for Life Sciences, National Laboratory Astana, Nazarbayev University. Z05H0P9, \\ Kabanbay Batyr Avenue 53, Block S1, Nur-Sultan, Kazakhstan. Tel. +7-717-2-06521, vemail: skozhakhmetov@nu.edu.kz \\ ${ }^{2}$ Kazakhstan Society of Researchers of Human Microbiome. Tawelsizdik 34, Z00T2C6 Nur-Sultan, Kazakhstan \\ ${ }^{3}$ Asfendiyarov Kazakh National Medical University. A05H2A6, Tole Bi Street 94, Almaty, Kazakhstan \\ ${ }^{4}$ Kazakh Medical University of Continuing Education Corporation. A15M0E6, Manasa Str. 34, Almaty, Kazakhstan \\ ${ }^{5}$ Ministry of Health of the Republic of Kazakhstan National Scientific Center of Phthisiopulmonology. A25H9G4, Bekkhozhin Str. 5, Almaty, \\ Kazakhstan \\ ${ }^{6}$ Innovative Center ArtScience. Z00T3X6, 23-15 Str. 11, Nur-Sultan, Kazakhstan \\ ${ }^{7}$ Medical University of Astana. Z10K9D8, Beibitshilik str. 49A, Nur-Sultan, Kazakhstan
}

Manuscript received: 20 May 2021. Revision accepted: 26 October 2021

\begin{abstract}
Kozhakhmetov S, Aitenov Y, Ramazanova B, Koloskova Y, Burkutbayeva T, Yeraliyeva L, Mustafina K, Beglarova G, Yergazina M, Kushugulova A. 2021. Influence of pneumococcal conjugate vaccine 13 on upper respiratory tract microbial biodiversity in infants. Biodiversitas 22: 5055-5060. Pneumococcal disease and its associated mortality are burdens on the healthcare system. The intensive introduction of pneumococcal vaccines has provided robust control and infection management worldwide. The Pneumococcal Conjugate Vaccine (PCV) has been successfully employed in the national programs of many countries. However, no studies have yet analyzed the effect of pneumococcal vaccines on Central Asian populations. We investigated the effect of the pneumococcal vaccine on the nasopharyngeal microbiome of infants under the age of two years. Samples were collected from healthy patients as part of routine hospital check-ups, then subjected to high-throughput sequencing of the V1-V3 region of 16S rRNA for bioinformatics analysis. The obtained data were combined with the results of a previously published study (with matching criteria) to increase statistical power. No significant differences were found in vaccination status, sex or age. Nevertheless, the results demonstrated structural changes in the microbiome of the upper respiratory tract under the influence of the PCV13 vaccine. The results of beta-diversity unweighted UniFrac distance measuring showed that the experimental groups differed in their qualitative (taxonomic) structure $(\mathrm{p}<0.1)$. In the vaccinated group, the abundance of several symbiotic taxa was significantly decreased, including Streptococcus pneumonia.
\end{abstract}

Keywords: Colonization, microbiome, Streptococcus pneumonia, upper respiratory tract, vaccination

\section{INTRODUCTION}

Streptococcus pneumonia (pneumococcus) is a pathogen generally found in the upper respiratory tract of a human. Pneumococcus usually colonizes the mucous membranes of the upper respiratory tract in early infancy, and it has been considered an asymptomatic commensal (carrier) in the nasopharynx (Tuomanen et al. 2004). Pneumococcal infection is generally caused by close contact with an infected person. According to an official report of the World Health Organization (WHO 2019), 1.6 billion human deaths are caused by pneumococcal diseases annually, including 0.7-1 billion children under the age of five years. These diseases are reported primarily in developing countries (Brouwer et al. 2010). For example, according to official statistics, in Kazakhstan, the incidence of pneumonia was 20.8 and 21.4 cases per 1,000 children in 2017 and 2018, respectively, with a mortality rate of 0.2\% (Kaydar et al. 2018; Seidullayeva et al. 2020). The frequency of diseases caused by pneumococcus is high among children aged between 1-23 months. In Pneumococcal
Meningitis (PM), mortality is several times higher than meningitis caused by meningococcal or hemophilic bacilli.

The use of the Pneumococcal Conjugate Vaccine (PCV) in the national immunization schedule has led to a significant decrease in the incidences of PM in countries with an active immunization program (Southern et al. 2018). Moore et al. (2015) reported a 64\% decrease in the incidence of PCV13-vaccinated children below the age of five years. Vaccination with PCV13 (Prevenar; few stages) began in 2012 in Kazakhstan (Government of the Republic of Kazakhstan 2009; Kuttykozhanova et al. 2014). The yearly percentage of vaccination coverage was: 2012: PCV1-51, PCV2-47, PCV3-39; 2015: PCV1-93, PCV2-85, PCV3-74; and 2018: PCV1-96, PCV2-94, PCV3-95.

Microflora has been thought to be directly involved in resisting colonization by incoming pathogens, the formation of the immune system, and the response of the macro-organism to infection (Ichinohe et al. 2011). The microflora of children under 24 months is still in the formative stage. Any interventions, including vaccination, can destabilize microflora homeostasis (Biesbroek et al. 
2014a, b). To date, few reports have described the effect of vaccines on the microflora of the upper respiratory tract. However, understanding whether changes occur in the structure of the microbiome is necessary since this may lead to susceptibility to respiratory diseases.

Therefore, this study aimed to scrutinize the effect of PCV13 on the composition and diversity of the structure of the bacterial community of the upper respiratory tract in children under two years.

\section{MATERIALS AND METHODS}

\section{Patients and study design}

The participants for the present study were selected and recruited in the city polyclinics of Almaty city (No. 12 and 31 ) and Karaganda city (No. 1, 2, 3, 4 and 5) for more than two years (2014-2016) as part of a project on monitoring Streptococcus pneumoniae strains in Kazakhstan and the effectiveness of anti-pneumococcal vaccination (Ramazanova et al. 2017b). The inclusion criteria were age and the absence of respiratory diseases at the time of check-up. In total, 39 children were recruited in Kazakhstan. The patient groups consisted of eight vaccinated and 31 unvaccinated patients aged between six and 24 months. To increase the statistical power by balancing the unequal sample size of the compared groups, an additional 50 patient samples matching the study criteria were retrieved from the PRJNA423191 study on PubMed (Kelly et al. 2017). The mean age of the vaccinated group was 9.34 months, and the mean age of the unvaccinated group was 9.92 months.

\section{Sample collection}

Nasopharyngeal (NP) samples were collected using the ESwab $^{\mathrm{TM}}$ Liquid Amies Collection and Transport System (Copan Diagnostics Inc., Murrieta, CA, USA; 482C). All the collected samples were kept in a freezer at $-80{ }^{\circ} \mathrm{C}$. For DNA extraction and subsequent analysis, samples were transported to the Centre for Life Sciences (Nazarbayev University, Nur-Sultan city) in compliance with the requirements for cold-chain delivery.

\section{Ethical approval}

The study was approved by the National Medical University Local Ethics Committee (Minutes No. 7 of June 30, 2014) and the Medical Ethics Committee (Minutes No. 19 of January 18, 2016) of the National Laboratory Astana at Nazarbayev University. Furthermore, a written informed consent signed by the parents of selected and recruited children to participate in the study was obtained for each participant. The study was conducted and monitored according to good clinical practice.

\section{DNA sequencing and post-processing}

DNA was isolated from swabs using the QIAamp DNA Mini Kit (Qiagen, 51306). The concentration of doublestranded DNA in isolated samples was determined using a Qubit 2.0 instrument and a Qubit dsDNA BR Assay kit (ThermoFisher, catalog number 32853). Following the manufacturer, the Library for Next-generation Sequencing was generated using a NEXTflex® 16S V1-V3 AmpliconSeq Kit (PerkinElmer, catalog number NOVA-4202-04) 's recommendations. The library quality was quantified by Qubit dsDNA HS Assay Kit with the Qubit 2.0 fluorometer system (Invitrogen, Life Technologies, Grand Island, NY, USA). Amplicons were sequenced on the MiSeq instrument (Illumina). The analysis was performed in $\mathrm{R}$ software (v.3.4.4; Anon 2018). The raw sequencing data were filtered by quality, cleaned and trimmed using the Divisive Amplicon Denoising Algorithm 2 package (DADA2, v.1.8.0; Callahan et al. 2016). DADA2 output consists of the exact immediate amplicon sequences (ASV) that replace the traditional OTUs produced by more 'traditional' pipelines, such as MOTHUR. Using DADA2 does not require rarefication of reads. Sequences were demultiplexed, primers were cut off, and high-quality filtering was performed on all sequences with a Phred index of less than $2(\mathrm{Q}<2)$ and a length of 150 bp for samples from Kazakhstan and 210 bp for samples from Kelly et al. (2017). After denoising and removing chimeras, the ASV table was constructed from all sequences using the collapseNoMismatch function in phyloseq (v1.28.0) to combine identical sequences up to shifts or length variation (McMurdie and Holmes 2013). After bimera removal, the taxonomic classification of the resulting sequence table was performed with the Ribosomal Database Project Naive Bayesian Classifier, using an $80 \%$ threshold filter as advised in the original publication (Wang et al. 2007). Only the ASV that represented $>0.005 \%$ of the total filtered were retained, as Bokulich et al. (2013) suggested. For analysis at the genus taxonomic level, ASVs were collapsed into phylum and genus ranks using the tax_glom function in phyloseq.

\section{Statistical analysis}

For alpha-diversity analysis of the abundance of the bacterial community, the Chaol and Ace indexes were used, and biodiversity measurement by employing the Shannon index. The non-parametric Mann-Whitney and Kruskal-Wallis tests compared two or more Shannon indexes. The Shapiro-Wilk normality test was used to analysis the distribution within samples. The determination of the influence of individual parameters such as sex, age and vaccination status on the relative biodiversity and abundance of taxa was calculated using the ANOSIM and PERMANOVA statistical tests on Bray-Curtis, UniFrac, and weighted and unweighted distances using the vegan package (v.2.5.3; Oksanen et al. 2012). The random dispersion affecting beta-diversity statistics was tested using beta-disper. All graphs were generated using ggplot2 (v.3.0.0; Wickham 2009).

\section{RESULTS AND DISCUSSION}

\section{Nasopharyngeal microbial communities}

A total of 89 NP specimens were used for microbial DNA sequencing analysis. The epidemiological characteristics of the study groups are shown in Table 1. After pre-processing pipeline preparation, the remaining amplicon sequences were 
assigned to 1,255 taxa across seven taxonomic ranks. Taxonomic classification revealed high inter-individual variability in the relative number of microbes at the genus level. The relative abundance of phyla and genera in the upper respiratory microbiota in vaccinated and unvaccinated children is presented in Figure 1.

The most abundant groups of microorganisms at the phylum and genus levels among all samples studied in the present study were determined. Among all bacterial microorganisms types, the highest occurrence was found for Firmicutes (46.43\%) followed by Proteobacteria (18.86\%), Actinobacteria (16.09\%) and Bacteroidetes (0.31\%), accounting for more than $80 \%$ of all identified phyla (Figure 1 , top panel). The 10 most represented genera of all identified taxa were the species of Staphylococcus, Streptococcus, Dolosigranulum, Lactobacillus, Haemophilus, Moraxella, Corynebacterium, Neisseria, Propionibacterium and Stenotrophormonas. All these bacteria represent the normal commensal flora of the healthy upper respiratory tract (Figure 1, lower panel). Mika et al. (2017) observed similar results on bacterial communities, where the most represented taxa were
Moraxellaceae, Streptococcaceae, Staphylococcaceae, Corynebacteriaceae and Pasteurellaceae. Similarly, Verhagen et al. (2021) also identified Moraxella sp., Corynebacterium propinquum, Dolosigranulum sp. and Streptococcus sp. as major bacterial taxa.

Several contradicting opinions exist on the effect of vaccines on the NP microbiome. Mika et al. (2017) showed that vaccination against $\mathrm{PCV}-13$ changed the taxonomic profile of the NP microbiome. According to them, the bacterial community, after vaccination, retained high biodiversity and low variability. Biesbroeck et al. (2014) found an increase in the absolute number of anaerobic bacteria such as Veillonella, Prevotella, Fusobacterium and Leptotrichia. In another study, Chaban et al. (2013) showed that the phylogenetic composition of the commensal microbiota consisted of Firmicutes (42.5\%), Proteobacteria (27.7\%), Actinobacteria (21.7\%), Bacteroides (5.5\%), fungi $(0.1 \%)$, human $(0.2 \%)$ and other bacterial taxa $(2.3 \%)$. Our results indicate that the NP profile characteristics differed from previously published data at the phylum level.

Table 1. Epidemiological characteristics of study groups

\begin{tabular}{lcccc}
\hline \multicolumn{1}{c}{ Variable } & Kazakhstan & Botswana & PCV13-vaccinated & Unvaccinated \\
\hline Patients (n) & 39 & 50 & 33 & 56 \\
Age, months (mean) & 12.12 & 7.82 & 9.34 & 9.92 \\
Age, months (Md) & 21.37 & 11.20 & 5.10 & 14.20 \\
Age, months (IQR) & 12.43 & 6.25 & 8.20 & 6.56 \\
Sex, male & 0.54 & 0.38 & 0.39 & 0.48 \\
\hline
\end{tabular}
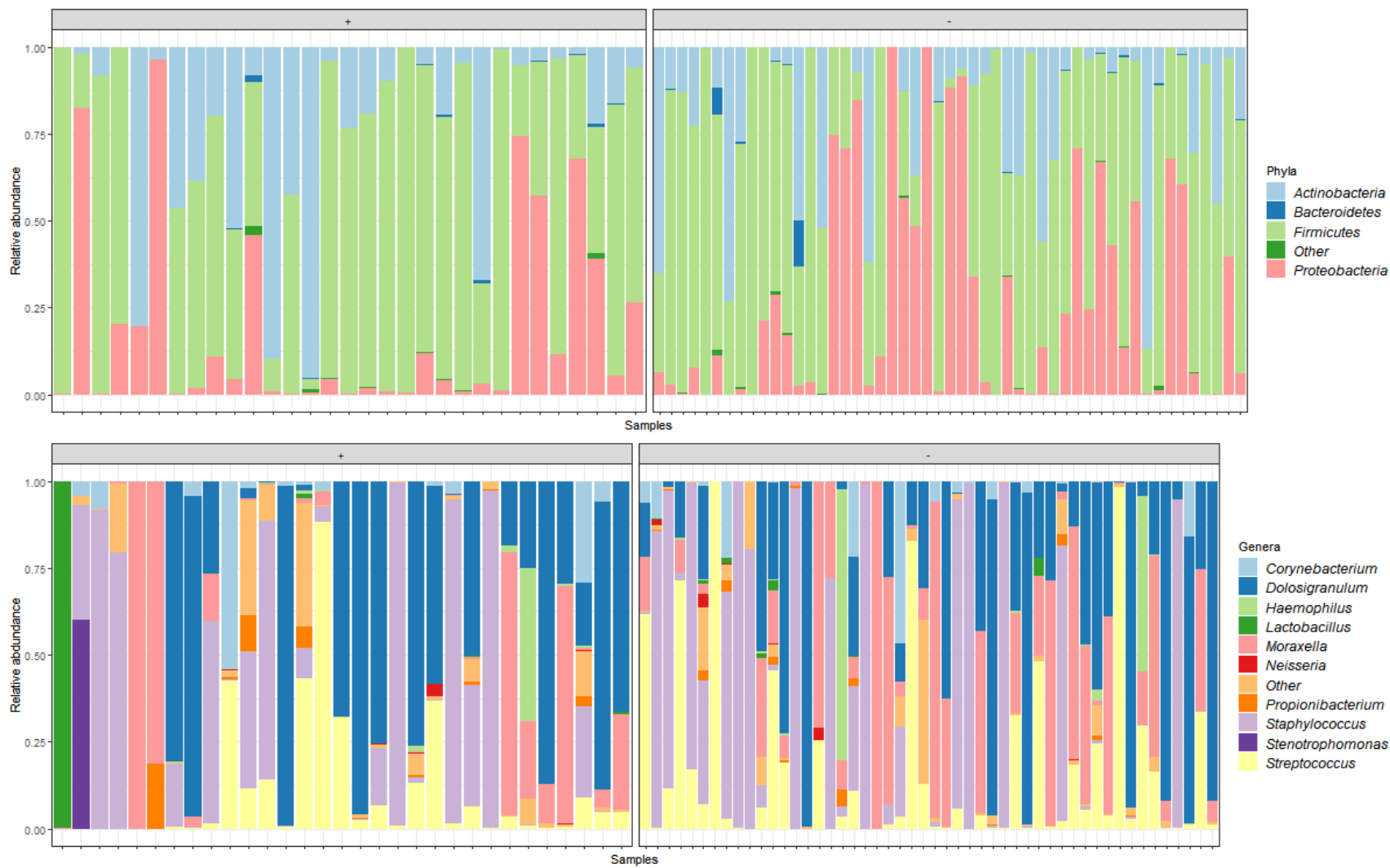

Figure 1. The most abundant taxa present in microbiome samples: top 4 phyla (upper panel) and top 10 genera (bottom panel). Taxa representing a minority $(<1 \%)$ are grouped in 'Other' 


\section{Effect of vaccination on community composition and structure}

The initial alpha- and beta-diversity analyses detected differences between vaccinated and unvaccinated patients (Shannon $p<0.01$,

Figure 3; PERMANOVA and ANOSIM tests on BrayCurtis, and weighted and unweighted UniFrac distances, data not shown).

Most of these differences disappeared when the samples taken from Kelly et al. (2017) were added for unified analysis. Nonetheless, the analysis of beta-diversity between groups using principal component analysis (PCoA) showed differences in the microbial structure of vaccinated and unvaccinated children, confirmed by unweighted UniFrac distance analysis (PERMANOVA $p<$ $0.1 *$, Figure 2).

\section{Effect of the country of origin on community composition and structure}

The impact of country origin (parameter) on microflora composition and structure was scrutinized using the alphadiversity Shannon test ( $p<0.001$, Figure 4$)$.

Beta-diversity analysis (UniFrac distances plotted on PCoA) also showed a statistical difference between samples on the country-of-origin parameter, both for unweighted (qualitative) and weighted (quantitative) measuring. However, the betadisper test showed a significant value, indicating that the difference could be partially attributed to the variation in dispersion (Figure 5).

\section{Effect of vaccination on NP microbial profiles}

We compared the mean relative abundances of the 10 most abundant genera in vaccinated and unvaccinated patients (Table 2). Six (Staphylococcus, Streptococcus, Moraxella, Dolosigranulum, Corynebacterium and

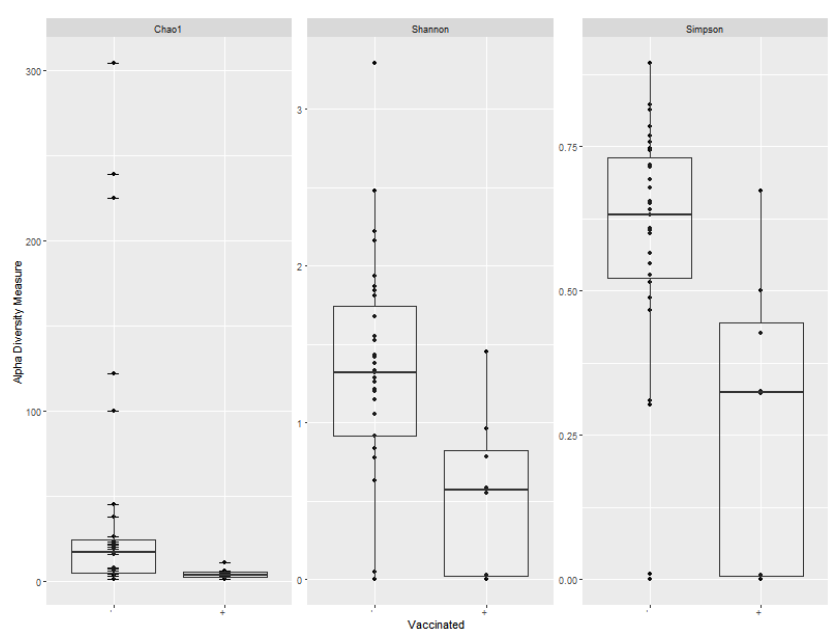

Figure 3. Alpha-diversity among Kazakhstani samples represented by Shannon, Chao1 and Simpson indices was significant for vaccination status $(p<0.01)$ but not for other parameters (data not shown)
Haemophilus) out of 10 genera in each category were the same, differing only in their mean relative abundance. However, a significant reduction in the relative abundance of Streptococcus was observed (-33\%). In addition, children with respiratory infections were less stable microbial profiles associated with Streptococcus and Haemophilus (Biesbroek et al. 2014a, b). In addition, the predominance of Moraxella, Streptococcus and Haemophilus were found associated with a range of disorders such as asthma and respiratory syncytial virus (Lang et al. 2018).

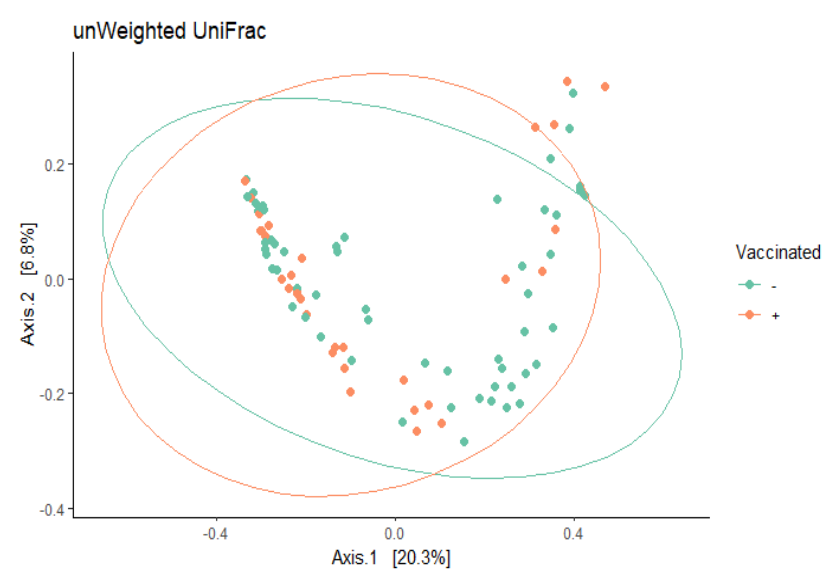

Figure 2. The beta-diversity between nasopharyngeal microbiota samples from vaccinated and unvaccinated groups was significant (PERMANOVA $p<0.1^{*}$, betadisper not significant). Ordination of the microbiome data used principal component analysis (PCoA) on weighted and unweighted UniFrac distances. Ellipses represent $95 \%$ confidence intervals

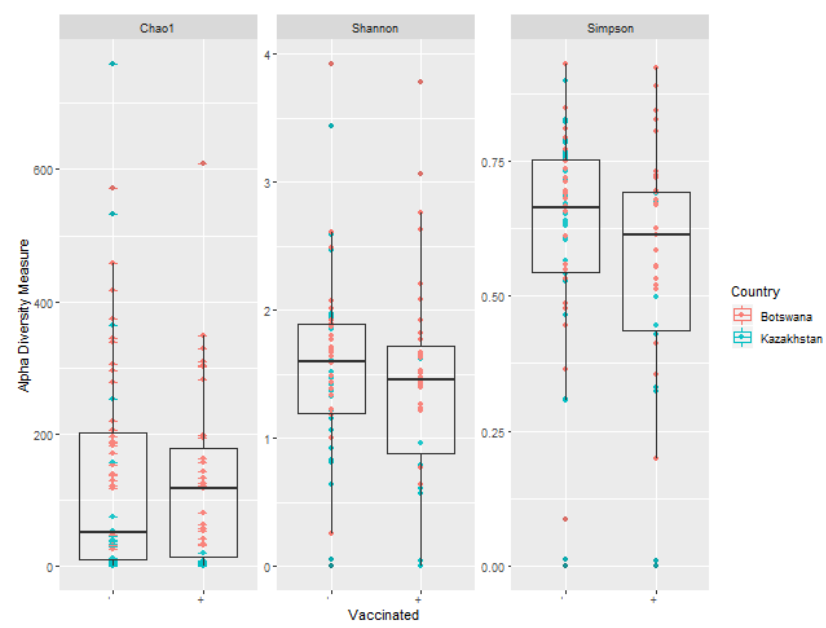

Figure 4. Alpha-diversity of NP microbiota samples from Kazakhstan. A significant difference was found using the Shannon index $(p<0.001)$ 

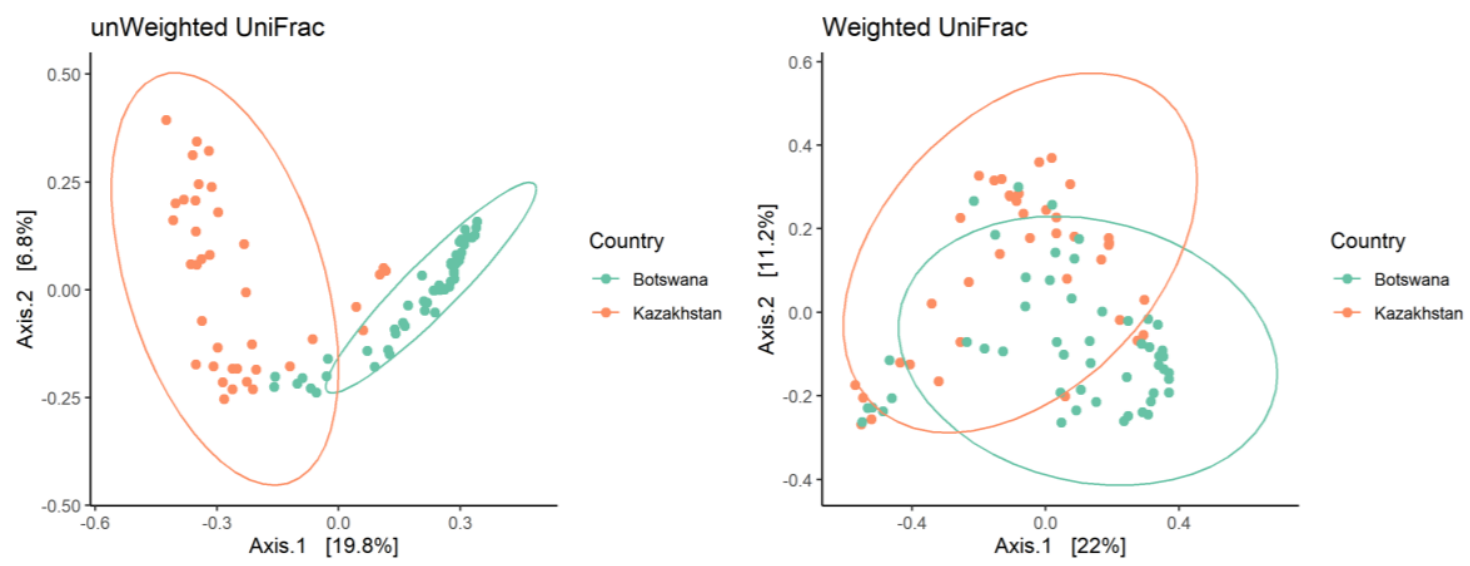

Figure 5. Beta-diversity between NP microbiota samples from different countries (PERMANOVA $\mathrm{p}<0.001$, betadisper $\mathrm{p}<0.05$ ). Ordination of the microbiome data using principal component analysis (PCoA) on weighted and unweighted UniFrac distances. Ellipses represent $0.95 \mathrm{CI}$

Table 2. Comparison of the relative abundance of taxa identified in unvaccinated (-) and vaccinated (+) samples. Six of the 10 most abundant genera were common between the two groups. A significant decrease in Streptococcus, Moraxella and Staphylococcus was observed in vaccinated patients

\begin{tabular}{|c|c|c|c|c|}
\hline Genus & $-(\%)$ & $+(\%)$ & Trend & $\begin{array}{c}\text { Change from } \\
\text { unvaccinated } \\
(\%)\end{array}$ \\
\hline Dolosigranulum & 28.18 & 35.46 & Up & 25.83 \\
\hline Staphylococcus & 27.50 & 26.23 & Down & -4.63 \\
\hline Moraxella & 19.95 & 14.07 & Down & -29.44 \\
\hline Streptococcus & 16.57 & 10.97 & Down & -33.80 \\
\hline Corynebacterium & 3.01 & 4.02 & Up & 33.73 \\
\hline Lactobacillus & Negligible & 3.44 & Up & \\
\hline Stenotrophomonas & Negligible & 2.02 & Up & \\
\hline Haemophilus & 2.76 & 1.73 & Down & -37.09 \\
\hline Propionibacterium & Negligible & 1.38 & $\mathrm{Up}$ & \\
\hline Acinetobacter & 1.05 & Negligible & Down & \\
\hline
\end{tabular}

Both of the aforementioned cases involved unvaccinated groups of children. Taken together with an increased mean relative abundance of Dolosigranulum and Corynebacterium (associated with breastfeeding; Biesbroek et al. 2014a,b), they may contribute to better resistance against pathogens. On the other hand, Moraxella, associated with a lower risk of respiratory infection (Biesbroek et al. 2014a,b), showed a reduced mean relative abundance in the vaccinated group (Table 2). This may indicate that further research is required to determine the specificity and efficiency of vaccines.

This is the first report on the NP microbiome in vaccinated and unvaccinated children in Central Asia to the best of our knowledge. The microbiome constituents at the genus taxonomic level were mainly consistent with the results of previous studies. However, the comparison between vaccinated and unvaccinated children revealed a significant change in the microbiome structure. Our results showed a dramatic reduction in the relative abundance of the genera Moraxella, Staphylococcus, Haemophilus and
Streptococcus resulting from the use of the vaccine. We also detected an increase in Corynebacterium and Dolosigranulum.

Infectious diseases of the upper respiratory tract severely impact health, especially in childhood, when the microbiome of this ecological niche is established. Our work has uncovered structural changes related to the reduction in the number of Streptococcus pneumonia and other commensal pathogens. Further studies on the development and variability of bacterial communities colonizing the upper respiratory tract may help scrutinize this primary barrier's role in upper respiratory disease.

\section{ACKNOWLEDGEMENTS}

The authors would like to thank the children and families who participated in the study. In addition, the authors are grateful to Dr. Matthew Kelly and colleagues for providing additional metadata from their study 'The nasopharyngeal microbiota of children with respiratory infections in Botswana'. This research was supported by the Ministry of Education and Science of the Republic of Kazakhstan within the framework of the project 'Health programming, the evolution of the infants' microbiome' (AP09259975).

\section{REFERENCES}

Anon. 2018. R Core Team, R: A language and environment for statistical computing. R Foundation for Statistical Computing, European Environment Agency, Vienna, Austria.

Biesbroek G, Bosch AATM, Wang X, Keijser BJF, Veenhoven RH, Sanders EAM, Bogaert D. 2014a. The impact of breastfeeding on nasopharyngeal microbial communities in infants. Am J Respir Crit Care Med 190 (3): 298-308. DOI: 10.1164/rccm.201401-0073OC.

Biesbroek G, Tsivtsivadze E, Sanders EA, Montijn R, Veenhoven RH, Keijser BJ, Bogaert D. 2014b. Early respiratory microbiota composition determines bacterial succession patterns and respiratory health in children. Am J Respir Crit Care Med 190 (11): 1283-1292. DOI: $10.1164 / \mathrm{rccm} .201407-12400 \mathrm{O}$. 
Bokulich NA, Subramanian S, Faith JJ, Gevers DG, Gordon JI, Knight R, Mills DA, Caporaso JG. 2013. Quality-filtering vastly improves diversity estimates from Illumina amplicon sequencing. Nat Methods 10 (1): 57-59. DOI: 10.1038/nmeth.2276.

Brouwer MC, Tunkel AR, van de Beek D. 2010. Epidemiology, diagnosis, and antimicrobial treatment of acute bacterial meningitis. Clin Microbiol Rev 23 (3): 467-492. DOI: 10.1128/CMR.00070-09.

Callahan B, McMurdie P, Rosen M, Han AW, Johnson AJA, Holmes SP. 2016. DADA2: High-resolution sample inference from Illumina amplicon data. Nat Methods 13(7): 581-583. DOI: 10.1038/nmeth.3869.

Chaban B, Albert A, Links MG, Gardy J, Tang P, Hill JE, Chaban B, Albert A, Links MG, Gardy J, Tang P, Hill JE. 2013. Characterization of the upper respiratory tract microbiomes of patients with pandemic H1N1 influenza. In: Tompkins SM (eds.). PLoS ONE 8 (7): e69559. DOI: 10.1371 journal.pone.0069559.

Government of the Republic of Kazakhstan. 2009. On approval of the list of diseases against which preventive vaccinations are carried out, rules of their conduct, and groups of the population subject to planned vaccination. Ministry of Health and Social Development of the Republic of Kazakhstan, Nursultan, Kazakhstan. [Russian]

Ichinohe T, Pang IK. Kumamoto Y, Peaper DR, Ho JH, Murray TS, Iwasaki A. 2011. Microbiota regulates immune defense against respiratory tract influenza a virus infection. Proc Natl Acad Sci USA 108 (13): 5354-5359. DOI: 10.1073/pnas.1019378108.

Kaydar EK, Kenesov AA, Yurchenko IV, Sabirov GS, Abdildina ZZ, Sabyrbaeva RA, Seisenbaev GT, Baymukhanova KH, Ponomareva SV, Ilyasov JR. 2018. Public health and activities of health organisations in Kazakhstan in 2017. Statistical compendium, Astana, Kazakhstan. [Russian]

Kelly MS, Surette MG, Smieja M, Pernica JM, Rossi L, Luinstra K, Steenhoff AP, Feemster KA, Goldfarb DM, Arscott-Mills T, Boiditswe S, Rulaganyang I, Muthoga C, Gaofiwe L, Mazhani T, Rawls JF, Cunningham CK, Shah SS, Seed PC. 2017. The nasopharyngeal microbiota of children with respiratory infections in Botswana. J Pediatr Infect Dis 36 (9): e211-e218. DOI 10.1097/INF.0000000000001607

Kuttykozhanova GG, Efendiyev IM, Baesheva DA. 2014. Clinical protocol for the diagnosis and treatment of meningococcal infections in children in the Republic of Kazakhstan, protocol. Ministry of Health and Social Development of the Republic of Kazakhstan, Nursultan, Kazakhstan. [Russian]

Lang A, Holt KE, Inouye M, Holt PG, Teo SM, Tang HHF, Judd LM, Lemanske RF, Evans MD, Jackson DJ, Gern JE. 2018 Nasopharyngeal microbiome during health and illness in early life. J Allergy Clin Immunol 141 (2): AB109. DOI 10.1016/j.jaci.2017.12.348.

McMurdie PJ, Holmes S. 2013. Phyloseq: An R package for reproducible interactive analysis and graphics of microbiome census data. PloS ONE 8 (4): e61217. DOI: 10.1371/journal.pone.0061217.
Mika M, Maurer J, Korten I, Allemann A, Aebi S, Brugger SD, Qi W, Frey U, Latzin P, Hilty M. 2017. Influence of the Pneumococcal Conjugate Vaccines on the temporal variation of pneumococcal carriage and the nasal microbiota in healthy infants: A longitudinal analysis of a case-control study. Microbiome 5 (1): 85. DOI: 10.1186/s40168-017-0302-6.

Moore MR, Link-Gelles R, Schaffner W, Lynfield R, Lexau C, Bennett NM, Petit S, Zansky SM, Harrison LH, Reingold A, Miller L, Scherzinger K, Thomas A, Farley MM, Zell ER, Taylor TH Jr, Pondo T, Rodgers L, McGee L, Beall B, Jorgensen JH WC. 2015. Effect of use of 13-Valent Pneumococcal Conjugate Vaccine in children on invasive pneumococcal disease in children and adults in the USA: Analysis of multisite, population-based surveillance. Lancet Infect Dis 15 (3): 301-309. DOI: 10.1016/S1473-3099(14)71081-3.

Oksanen J, Blanchet FG, Friendly M, Kindt R, Legendre P, McGlinn D, Minchin PR, O'Hara RB, Simpson GL, Solymos P, Stevens MHH, Szoecs E, Wagner H. 2012. Vegan: Community Ecology Package. R Package Version 2.2-0. http://CRAN.Rproject.org/package=vegan.

Ramazanova BA, Eralieva LT, Mustafina KK, Koloskova EA. 2017b. Approaches to the implementation of the first research project on the study of pneumococcal infection in Kazakhstan. Med Almaty 3: 2-6. [Russian]

Seidullayeva A, Bayesheva D, Turdalina B, Altynbekova A, Utegenova R, Nurtazina G, Volkova G, Zhuzzhasarova A, Omarova A, Elubayeva A, Kozhakhmetov S. 2020. Sensorineural hearing loss after bacterial meningitis in children residing in Nur-Sultan. Infektsionnye Bolezni 18 (4): 189-194. DOI: 10.20953/1729-9225-2020-4-189-194. [Russian]

Southern J, Andrews N, Sandu P, Sheppard CL, Waight PA, Fry NK, van Hoek AJ, Miller E. 2014. Pneumococcal carriage in children and adults two years after introduction of the thirteen Valent Pneumococcal Conjugate Vaccine in England. Vaccine 32 (34): 43494355. DOI: 10.1016/j.vaccine.2014.03.017.

Tuomanen EI, Mitchell TJ, Morrison D, Spratt BG (eds.). 2004. The Pneumococcus. American Society of Microbiology, Washington DC. DOI: $10.1128 / 9781555816537$.

Verhagen LM, Rivera-Olivero IA, Clerc M, Chu MLJN, van Engelsdorp Gastelaars J, Kristensen MI, Berbers GAM, Hermans PWM, de Jonge MI, de Waard JH, Bogaert D. 2021. Nasopharyngeal microbiota profiles in rural Venezuelan children are associated with respiratory and gastrointestinal infections. Clin Infect Dis 72 (2): 212-221. DOI: 10.1093/cid/ciaa015.

Wang Q, Garrity GM, Tiedje JM, Cole JR. 2007. Naive Bayesian Classifier for rapid assignment of rRNA Sequences into the new bacterial taxonomy. Appl Environ Microbiol 73 (16): 5261-5267. DOI: 10.1128/AEM.00062-07.

WHO. 2019. Routine immunization country profile - Kazakhstan. WHO/UNICEF Joint Reporting Form, Rome.

Wickham H. 2009. ggplot2: Elegant Graphics for Data Analysis, SpringerVerlag. Springer, New York, NY. DOI: 10.1007/978-0-387-98141-3 